\title{
Association Between Systemic Corticosteroid Therapy and Mortality in Critically III Patients with Acute Exacerbation of Chronic Obstructive Pulmonary Disease: A Retrospective Cohort Study
}

\section{Tingyu Pan}

Nanjing University of Chinese Medicine https://orcid.org/0000-0003-1005-5964

\section{Le Bai}

Nanjing University of Chinese Medicine

\section{Shi Chen}

Affiliated Hospital of Nanjing University of Chinese Medicine: Jiangsu Province Academy of Traditional Chinese Medicine

\section{Kejie Zhou}

Shanghai Jiaotong University: Shanghai Jiao Tong University

\section{Jing Wang}

Nanjing University of Chinese Medicine

Yehui Li

Nanjing University of Chinese Medicine

\section{Weizhou Zhang}

Nanjing University of Chinese Medicine

Xianmei Zhou ( $\nabla$ zhouxianmeijs@aliyun.com )

Jiangsu Province Hospital of Chinese Medicine https://orcid.org/0000-0001-7662-2306

\section{Hailang He}

Affiliated Hospital of Nanjing University of Chinese Medicine: Jiangsu Province Academy of Traditional Chinese Medicine

\section{Research}

Keywords: chronic obstructive pulmonary disease, corticosteroids, exacerbation, eosinophil, critical care, mortality

Posted Date: June 11th, 2021

DOI: https://doi.org/10.21203/rs.3.rs-571219/v1 
License: (c) (i) This work is licensed under a Creative Commons Attribution 4.0 International License. Read Full License 


\section{Abstract \\ Background}

Whether the patients admitted in intensive care unit (ICU) due to acute exacerbation of chronic obstructive pulmonary disease (AECOPD) would benefit from systemic corticosteroid treatment remains controversial. In this study, we aim to investigate the relationship between the systemic corticosteroid treatment and mortality in patients with severe AECOPD, using the public Medical Information Mart for Intensive Care III database (MIMIC III).

\section{Methods}

The baseline characteristics, laboratory examination and prognosis of AECOPD patients were retrieved from MIMIC III. Multivariate logistic regression was used to analyze the association between systemic corticosteroid treatment and prognosis. Propensity score matching (PSM) was employed to validate our findings.

\section{Results}

A total of 882 patients were included in our study, of which 293 patients (33.22\%) underwent systemic glucocorticoid (GC) treatment. 30-day mortality was not reduced by treatment with systemic corticosteroid in this study. The adjusted odds ratio (OR) was 1.296 (95\% Cl: $0.907-1.852, p=0.155)$ and $1.243(95 \% \mathrm{Cl}: 0.867-1.782, \mathrm{p}=0.236)$ in the logistic regression model 1 and model 2 respectively. Similar results were replicated after PSM (OR 1.144, 95\% Cl: $0.744-1.759, p=0.541$ ). In the subgroup of patients with initial eosinophil concentration $\geq 0.35 \%$, corticosteroid treatment resulted in worse outcomes compared with non-GC group. The adjusted odds ratio of 30-day mortality and in-hospital mortality for glucocorticoid therapy were 1.691 (95\% Cl: $1.002-2.855, \mathrm{p}=0.049)$ and $2.247(95 \% \mathrm{Cl}$ : $1.218-4.147, p=0.010$ ) respectively.

\section{Conclusion}

Our study observed that Systemic corticosteroid therapy did not improve the mortality of severe AECOPD patients in ICU and aggravated the mortality of patients with high eosinophils. The results need to be verified in randomized controlled trials.

\section{Background}

Chronic obstructive pulmonary disease (COPD) is one of the top three causes of death worldwide [1]. Exacerbations play an important role in COPD progression and accelerate the decline in lung function of the patients, resulting in reduced physical activity and an increased risk of death [2-4]. Treatments for 
acute exacerbation of chronic obstructive pulmonary disease (AECOPD) includes antibiotics, bronchodilators, corticosteroids and respiratory support (https://goldcopd.org/2021-gold-reports/).

The best available evidence on the efficacy of systemic corticosteroid therapy on the outcomes of AECOPD is summarized by Walters et al. in a cochrane meta-analysis of randomized controlled trials which contained sixteen studies [5]. It found that patients treated with systemic corticosteroids either injections or tablets compared with dummy treatment were less likely to experience treatment failure (122 fewer people per 1000 treated) with high quality evidence but corticosteroid treatment did not reduce the mortality up to 30 days [5]. However, most of the studies with regard to systemic corticosteroids for AECOPD excluded people who had signs of a very severe exacerbation or consistent with significant comorbidity. Only two of them [6, 7] involved the patients mechanically ventilated or admitted to the intensive care unit (ICU). The results of these two studies were contradictory to some extent. The multicenter Spanish randomized study by Alia et al. included 83 patients and showed that a 10-day course of intravenous methylprednisolone treatment reduced the duration of mechanical ventilation by 1 day and the rate of noninvasive ventilation (NIV) failure ( $0 \%$ vs $37 \% ; p=0.004)$ [6]. The multicenter Tunisian randomized study by Abroug et al. included 217 patients and showed that a 10-day course of oral prednisone treatment didn't reduce the risk of NIV (relative risk $1.25,95 \% \mathrm{Cl} 0.56-2.8 ; \mathrm{p}=0.59$ ), as well as the median mechanical ventilation duration and intensive care unit length of stay [7]. Meanwhile, steroid treatment had no impact on ICU mortality and induced an increased risk of hyperglycaemic episodes in these two studies [6, 7]. Both of these two studies were stopped before their completion of the planned sample size because of a slow inclusion rate.

To date, whether the benefit from systemic corticosteroids to AECOPD patients obtained from non-ICU studies could promote to critically ill patients in ICU is uncertain. This study aims to evaluate the associations between the use of systemic corticosteroids and mortality in severely ill patients with AECOPD, and to explore whether the associations varied between different types of AECOPD, such as eosinophil (EOS) typing.

\section{Methods}

\section{Study Design and Data Source}

This study used a retrospective cohort design based on a publicly available critical care database called the Medical Information Mart for Intensive Care III (MIMIC-III) [8]. The MIMIC-III (v1.4) database contains 58976 admissions of 46520 patients at the Beth Israel Deaconess Medical Center between 2001 and 2012. One author (TYP) obtained access to the database and was responsible for data extraction. Since MIMIC-III project was approved by the Institutional Review Boards of Beth Israel Deaconess Medical Center (Boston, MA) and the Massachusetts Institute of Technology (Cambridge, MA), institutional review board (IRB) approval from our institution was exempted.

\section{Data Extraction and Participants}


Data extraction was performed on BigQuery and reference codes could be accessed from the MIMIC Code Repository [9] (https://github.com/MIT-LCP/mimiccode). We obtained the related information on patients who were diagnosed as AECOPD on discharge using AECOPD ICD-9-CM code 491.21. The patients with missing data (basic information or blood eosinophil concentrations) were excluded. The definition of AECOPD was episodes of worsening of respiratory symptoms that results in additional therapy [10]. Eosinophil initial was defined es the first record value of blood eosinophil concentrations of each hospital admission. Eosinophil max was defined as the maximum value during the hospitalization and eosinophil mean the arithmetic mean of all values during hospitalization. Some patients had multiple admissions to hospital. For these patients, only the most recent hospital was used [11]. The detailed process of data extraction is shown in Figure 1. We collected the following variables for the first day of ICU admission: age at the time of hospital admission, gender, admission type, sequential organ failure assessment (SOFA) score [12], simplified acute physiology score II (SAPS II) [13] and systemic inflammatory response syndrome (SIRS) score [14]. Severity of illness scores were from the materialized views generated by the code from the github website (https://github.com/MIT-LCP/mimic-code/tree/master/concepts) and were calculated based on the data obtained within the first $24 \mathrm{~h}$ of each patient's ICU's stay. Comorbidities in categories proposed by Elixhauser et al. [15] used an algorithm by Quan et al. [16].

All the prescription records of systemic glucocorticoids (GC) are extracted and then merged into the patient information of AECOPD. In brief, the codes provided in Supplementary material 1 was used to extract the glucocorticoid prescription records from table PRESCRIPTION. Then, systemic glucocorticoid prescription records for patients diagnosed with AECOPD was extracted and merged into the basic information table. Cream, eye drops, suppository, inhalation and nasal spray types of glucocorticoids are excluded.

\section{Outcomes}

The primary outcome was 30-day mortality. The secondary outcomes included in-hospital mortality and hospital length of stay (LOS). Since a patient might have more than one admission, hospital mortality and length of hospital stay were determined only by the recent hospital admission. For patients who were discharged alive, the date of death was from the social security database. The hospital LOS was stratified into two levels based on the median: hospital LOS level 1 ( $\leq 8$ days) and level 2 (>8 days).

\section{Statistical Analysis}

Data were described as median (25th-75th percentiles), or numbers (percentages) according to types of variables. Univariate analysis was carried out between survivors and non-survivors, systemic corticosteroid treatment group and non-systemic corticosteroid treatment group to screen variables associated with 30-day mortality and systemic corticosteroid usage. The Wilcoxon rank-sum test was performed for the continuous variable comparisons between groups. Comparisons for categorical variables were made using the chi-square test or Fisher's exact test as appropriate. 
The logistic regression models were selected to explore the relationship between systemic corticosteroid therapy and outcomes. Potential confounders were chosen based on univariate analysis and clinical experience. Two multivariate modes were used to adjust potential confounders: (1) model 1, variables were adjusted for age (years), Elixhauser comorbidity score, SAPS II, SIRS, invasive mechanical ventilation, and initial value of eosinophil concentration; (2) model 2, the same as model 1, but SAPS II was replaced with SOFA and the Elixhauser comorbidity score replaced with specific comorbidities, including cardiac arrhythmias, liver disease, metastatic cancer, solid tumor, coagulopathy, obesity, drug abuse and psychoses.

Subgroup analysis was performed by restricting to patients with different EOS initial concentration. Two cut-off values were tried (EOS initial concentration $<0.35 \%$ or $\geq 0.35 \%$, EOS initial concentration $<2 \%$ or $\geq 2 \%$ ).

Relationship between the route of administration of systemic corticosteroids and outcomes was explored by categorizing GC group according to administration route (intravenously, orally, or combination of intravenous and oral administration).

Propensity score matching (PSM) was employed to validate our findings. Propensity score (PS) measures the probability of a patient being assigned to systemic GC treatment. The propensity score for an individual was estimated based on the given covariates of SOFA score, SAPS II score, SIRS score, eosinophil initial concentration, congestive heart failure, peripheral vascular disease, hypertension, other neurologic disorder, hypothyroidism, obesity, blood loss anemia, depression using a logistic regression model. One-to-one nearest neighbor matching with a calliper width of 0.02 was applied in the present study. P-values between the and the GC cohort and non-GC cohort were calculated to assess the balance between groups after matching. Logistic regression was then performed to adjust for residual imbalance by including parameters with $p<0.05$ and potential confounders judged by clinical expertise.

Data analyses were performed by STATA 15.1 and SPSS 22 software; a two-tailed $p<0.05$ was considered statistically significant.

\section{Results}

\section{Baseline Characteristics and Outcomes}

MIMIC-III contained 943 unique subjects with 1,198 hospital admissions due to AECOPD, and a total of 882 patients were included finally in our cohort. The baseline characteristics of included patients are summarized in Table 1. As expected, survivors in 30-day had lower Elixhauser comorbidity scores (median 10 vs. 14, p < 0.001), SOFA scores (median 3 vs. $5, \mathrm{p}<0.001$ ), and SAPS II score (median 35vs. $45, p<0.001)$ than non-survivors. Variables of eosinophils were significantly higher for survivors than non-survivors in 30 days. The use of invasive mechanical ventilation was significantly more frequent in the non-survivor group and less systemic corticosteroid treatment was observed for the survivor group [211 (30.94\%) vs. $82(41.00 \%) ; p=0.008]$. 
Page $7 / 25$ 
Table 1

Comparisons of Baseline Characteristics Between Survivors and Non-Survivors

\begin{tabular}{|c|c|c|c|c|}
\hline Variable & $\begin{array}{l}\text { Survivors } \\
(n=682)\end{array}$ & $\begin{array}{l}\text { Non-Survivors } \\
(n=200)\end{array}$ & $\begin{array}{l}\text { Total } \\
(\mathrm{n}=882)\end{array}$ & $\mathbf{P}$ \\
\hline Gender, male, n (\%) & $337(49.41)$ & $98(49.00)$ & 435 (49.32) & 0.918 \\
\hline Admission age [median (IQR)] & $72(65,80)$ & $79(72,84)$ & $74(66,81)$ & $<0.001$ \\
\hline Admission type, $\mathrm{n}(\%)$ & & & & 0.102 \\
\hline Elective & $15(2.20)$ & $1(0.50)$ & $16(1.81)$ & \\
\hline Emergency & $659(96.63)$ & $194(97.00)$ & $853(96.71)$ & \\
\hline Urgent & $8(1.17)$ & $5(2.50)$ & $13(1.47)$ & \\
\hline Admission period, $\mathrm{n}(\%)$ & & & & 0.094 \\
\hline Before 2008 & $336(49.27)$ & $112(56.00)$ & $448(50.79)$ & \\
\hline $2008-2012$ & $346(50.73)$ & $88(44.00)$ & $434(49.21)$ & \\
\hline $\begin{array}{l}\text { Elixhauser comorbidity score [median } \\
(\text { IQR)] }\end{array}$ & $10(6,16)$ & $14(9,19)$ & $11.5(7,17)$ & $<0.001$ \\
\hline \multicolumn{5}{|l|}{ Comorbidities, n (\%) } \\
\hline Congestive Heart Failure & $368(53.96)$ & $113(56.50)$ & $481(54.54)$ & 0.526 \\
\hline Arrhythmia & $276(40.47)$ & $115(57.50)$ & $391(44.33)$ & $<0.001$ \\
\hline Valve Disorder & $87(12.76)$ & $35(17.50)$ & $122(13.83)$ & 0.088 \\
\hline Pulmonary Circulation Disorder & $100(14.66)$ & $21(10.50)$ & $121(13.72)$ & 0.132 \\
\hline Peripheral Vascular Disease & $65(9.53)$ & $21(10.50)$ & $86(9.75)$ & 0.685 \\
\hline Hypertension & $400(58.65)$ & $109(54.50)$ & $509(57.71)$ & 0.296 \\
\hline Paralysis & $14(2.05)$ & $2(1.00)$ & $16(1.81)$ & 0.546 \\
\hline Other neurologic disorder & $64(9.38)$ & $25(12.50)$ & $89(10.09)$ & 0.198 \\
\hline Diabetes & $169(24.78)$ & $43(21.50)$ & $212(24.04)$ & 0.340 \\
\hline Diabetes with Complications & $36(5.28)$ & $8(4.00)$ & $44(4.99)$ & 0.465 \\
\hline Hypothyroidism & $79(11.58)$ & $21(10.50)$ & $100(11.34)$ & 0.671 \\
\hline Renal Failure & $125(18.33)$ & $41(20.50)$ & $166(18.82)$ & 0.490 \\
\hline Liver Disease & $34(4.99)$ & $21(10.50)$ & $55(6.24)$ & 0.005 \\
\hline Ulcer & $7(1.03)$ & $3(1.50)$ & $10(1.13)$ & 0.703 \\
\hline
\end{tabular}




\begin{tabular}{|c|c|c|c|c|}
\hline Variable & $\begin{array}{l}\text { Survivors } \\
(n=682)\end{array}$ & $\begin{array}{l}\text { Non-Survivors } \\
(n=200)\end{array}$ & $\begin{array}{l}\text { Total } \\
(\mathrm{n}=\mathbf{8 8 2})\end{array}$ & $\mathbf{P}$ \\
\hline AIDS & $4(0.59)$ & $1(0.50)$ & $5(0.57)$ & 1.000 \\
\hline Lymphoma & $9(1.32)$ & $5(2.50)$ & $14(1.59)$ & 0.330 \\
\hline Cancer Metastases & $26(3.81)$ & $20(10.00)$ & $46(5.22)$ & 0.001 \\
\hline Solid Tumor & $28(4.11)$ & $17(8.50)$ & $45(5.10)$ & 0.013 \\
\hline Rheumatoid Arthritis & $27(3.96)$ & $6(3.00)$ & 33 (3.74) & 0.530 \\
\hline Coagulopathy & $47(6.89)$ & $28(14.00)$ & $75(8.50)$ & 0.002 \\
\hline Obesity & $61(8.94)$ & $9(4.50)$ & $70(7.94)$ & 0.041 \\
\hline Weight Loss & $33(4.84)$ & $16(8.00)$ & $49(5.56)$ & 0.086 \\
\hline Fluid and Electrolyte Disorder & $310(45.45)$ & $103(51.50)$ & $413(46.83)$ & 0.132 \\
\hline Blood Loss Anemia & $22(3.23)$ & $6(3.00)$ & $28(3.17)$ & 0.873 \\
\hline Deficiency Anemia & $35(5.13)$ & $7(3.50)$ & $42(4.76)$ & 0.341 \\
\hline Alcohol Abuse & $50(7.33)$ & $11(5.50)$ & $61(6.92)$ & 0.369 \\
\hline Drug Abuse & $36(5.28)$ & $2(1.00)$ & $38(4.31)$ & 0.009 \\
\hline Psychoses & $37(53.96)$ & $3(56.50)$ & $40(54.54)$ & 0.019 \\
\hline Depression & $103(15.10)$ & $22(11.00)$ & $125(14.17)$ & 0.144 \\
\hline \multicolumn{5}{|l|}{ Severity of illness [median (IQR)] } \\
\hline SOFA score & $3(2,5)$ & $5(3,7)$ & $4(2,6)$ & $<0.001$ \\
\hline SAPS II score & $35(29,43)$ & $45(36,65)$ & $37(30,46)$ & $<0.001$ \\
\hline SIRS score & $3(2,3)$ & $3(3,4)$ & $3(2,4)$ & 0.001 \\
\hline Invasive mechanical ventilation, $\mathrm{n}(\%)$ & $262(38.24)$ & $106(53.00)$ & $368(41.72)$ & $<0.001$ \\
\hline \multicolumn{5}{|l|}{ Eosinophils, \% [median (IQR)] } \\
\hline Eosinophil initial & $\begin{array}{l}0.5 \\
(0.1,1.5)\end{array}$ & $0.2(0,1.0)$ & $\begin{array}{l}0.4 \\
(0.1,1.3)\end{array}$ & $<0.001$ \\
\hline Eosinophil max & $\begin{array}{l}1.0 \\
(0.3,2.2)\end{array}$ & $0.7(0.15,1.4)$ & $\begin{array}{l}1.0 \\
(0.3,2.0)\end{array}$ & $<0.001$ \\
\hline Eosinophil mean & $\begin{array}{l}0.6 \\
(0.2,1.4)\end{array}$ & $\begin{array}{l}0.358 \\
(0.1,1.0)\end{array}$ & $\begin{array}{l}0.5 \\
(0.2,1.3)\end{array}$ & $<0.001$ \\
\hline Hospital LOS, day [median (IQR)] & $8(5,14)$ & $8(4,13)$ & $8(5,14)$ & 0.015 \\
\hline
\end{tabular}




\begin{tabular}{|lllll|}
\hline Variable & $\begin{array}{l}\text { Survivors } \\
(\mathbf{n = 6 8 2})\end{array}$ & $\begin{array}{l}\text { Non-Survivors } \\
(\mathbf{n = 2 0 0 )}\end{array}$ & $\begin{array}{l}\text { Total } \\
(\mathbf{n = 8 8 2})\end{array}$ & $\mathbf{P}$ \\
\hline Systemic corticosteroids treatment, $\mathrm{n}(\%)$ & $211(30.94)$ & $82(41.00)$ & $293(33.22)$ & 0.008 \\
\hline
\end{tabular}

IQR interquartile range, SOFA sequential organ failure assessment, SAPSII Simplified Acute Physiology Score II, SIRS systemic inflammatory response syndrome, LOS length of stay

Of the study cohort, 293 patients had been exposed to systemic glucocorticoid treatment, and the remaining 589 patients did not have prescription records for systemic glucocorticoids. Table 2 shows the baseline characteristics for the GC and non-GC groups before and after propensity score matching. In general, patients in the GC group were more critically ill than the non-GC group, with significantly higher SOFA score (median 4 vs. 3; $p=0.002$ ) and SAPS II score (median 40 vs. 37; $p=0.009$ ). Patients in the GC group were more likely to be enrolled during 2000-2008. Admissions times due to AECOPD were balanced between the two groups. 
Table 2

Baseline characteristics comparing the GC group and non-GC group before and after propensity score matching

\begin{tabular}{|c|c|c|c|c|c|c|}
\hline \multirow[t]{2}{*}{ Variable } & \multicolumn{2}{|c|}{ Before matching } & \multicolumn{2}{|c|}{ After matching } & \multicolumn{2}{|l|}{$\mathbf{P}$} \\
\hline & $\begin{array}{l}\text { Non-GC }(\mathrm{n} \\
=589)\end{array}$ & $\begin{array}{l}\mathrm{GC}(\mathrm{n}= \\
293)\end{array}$ & $\begin{array}{l}\text { Non-GC }(\mathrm{n} \\
=258)\end{array}$ & $\begin{array}{l}\mathrm{GC}(\mathrm{n}= \\
258)\end{array}$ & Before & After \\
\hline Gender, male, n (\%) & $\begin{array}{l}291 \\
(49.41)\end{array}$ & $\begin{array}{l}144 \\
(49.15)\end{array}$ & $\begin{array}{l}128 \\
(49.61)\end{array}$ & $\begin{array}{l}125 \\
(48.45)\end{array}$ & 0.942 & 0.792 \\
\hline $\begin{array}{l}\text { Admission age [median } \\
(\text { IQR)] }\end{array}$ & $74(66,82)$ & $\begin{array}{l}75(67 \\
80)\end{array}$ & $\begin{array}{l}73,5(65 \\
82)\end{array}$ & $\begin{array}{l}75(67 \\
80)\end{array}$ & 0.790 & 0.570 \\
\hline Admission type, $\mathrm{n}(\%)$ & & & & & 0.408 & 0.071 \\
\hline Elective & $13(2.21)$ & $3(1.02)$ & $5(1.94)$ & $3(1.16)$ & & \\
\hline Emergency & $\begin{array}{l}556 \\
(96.10)\end{array}$ & $\begin{array}{l}287 \\
(97.95)\end{array}$ & $\begin{array}{l}246 \\
(95.35)\end{array}$ & $43(254)$ & & \\
\hline Urgent & $10(1.70)$ & $3(1.02)$ & $7(2,71)$ & $1(0.39)$ & & \\
\hline Admission period, $\mathrm{n}(\%)$ & & & & & $\dot{0} 001$ & $<$. \\
\hline Before 2008 & $\begin{array}{l}198 \\
(33.62)\end{array}$ & $\begin{array}{l}250 \\
(85.32)\end{array}$ & $\begin{array}{l}160 \\
(62.02)\end{array}$ & $\begin{array}{l}221 \\
(85.66)\end{array}$ & & \\
\hline $2008-2012$ & $\begin{array}{l}391 \\
(66.38)\end{array}$ & $\begin{array}{l}43 \\
(14.68)\end{array}$ & 98 (37.98) & $\begin{array}{l}37 \\
(14.34)\end{array}$ & & \\
\hline $\begin{array}{l}\text { Elixhauser comorbidity } \\
\text { score [median (IQR)] }\end{array}$ & $11(5,17)$ & $\begin{array}{l}12 \\
(8,16)\end{array}$ & $11.5(7,16)$ & $\begin{array}{l}12(8, \\
16)\end{array}$ & 0.197 & 0.633 \\
\hline \multicolumn{7}{|l|}{ Comorbidities, n (\%) } \\
\hline Congestive Heart Failure & $\begin{array}{l}305 \\
(51.78)\end{array}$ & $\begin{array}{l}176 \\
(60.07)\end{array}$ & $\begin{array}{l}145 \\
(56.20)\end{array}$ & $\begin{array}{l}146 \\
(56.59)\end{array}$ & 0.020 & 0.929 \\
\hline Arrhythmia & $\begin{array}{l}264 \\
(44.82)\end{array}$ & $\begin{array}{l}127 \\
(43.34)\end{array}$ & $\begin{array}{l}113 \\
(43.80)\end{array}$ & $\begin{array}{l}109 \\
(42.25)\end{array}$ & 0.677 & 0.722 \\
\hline Valve Disorder & $85(14.43)$ & $\begin{array}{l}37 \\
(12.63)\end{array}$ & $32(12.40)$ & $\begin{array}{l}32 \\
(12.40)\end{array}$ & 0.465 & 1.000 \\
\hline $\begin{array}{l}\text { Pulmonary Circulation } \\
\text { Disorder }\end{array}$ & $88(14.94)$ & $\begin{array}{l}33 \\
(11.26)\end{array}$ & $38(14.73)$ & $\begin{array}{l}29 \\
(11.24)\end{array}$ & 0.135 & 0.239 \\
\hline Peripheral Vascular Disease & $69(11.71)$ & $\begin{array}{l}17 \\
(5.80)\end{array}$ & $17(6.59)$ & $\begin{array}{l}17 \\
(6.59)\end{array}$ & 0.005 & 1.000 \\
\hline Hypertension & $\begin{array}{l}368 \\
(62.48)\end{array}$ & $\begin{array}{l}141 \\
(48.12)\end{array}$ & $\begin{array}{l}127 \\
(49.22)\end{array}$ & $\begin{array}{l}135 \\
(52.33)\end{array}$ & $\dot{0} 001$ & 0.481 \\
\hline Paralysis & $13(2.21)$ & $3(1.02)$ & $3(1.16)$ & $2(0.78)$ & 0.215 & 1.000 \\
\hline
\end{tabular}




\begin{tabular}{|c|c|c|c|c|c|c|}
\hline \multirow{2}{*}{$\begin{array}{l}\text { Variable } \\
\text { Other neurologic disorder }\end{array}$} & \multicolumn{2}{|c|}{ Before matching } & \multicolumn{2}{|c|}{ After matching } & \multicolumn{2}{|l|}{$\mathbf{P}$} \\
\hline & $69(11.71)$ & $\begin{array}{l}20 \\
(6.83)\end{array}$ & $13(5.04)$ & $\begin{array}{l}19 \\
(7.36)\end{array}$ & 0.023 & 0.273 \\
\hline Diabetes & $\begin{array}{l}150 \\
(25.47)\end{array}$ & $\begin{array}{l}62 \\
(21.16)\end{array}$ & $66(25.58)$ & $\begin{array}{l}56 \\
(21.71)\end{array}$ & 0.519 & 0.300 \\
\hline $\begin{array}{l}\text { Diabetes with } \\
\text { Complications }\end{array}$ & $31(5.26)$ & $\begin{array}{l}13 \\
(4.44)\end{array}$ & $9(3.49)$ & $\begin{array}{l}12 \\
(4.65)\end{array}$ & 0.595 & 0.504 \\
\hline Hypothyroidism & 78 (13.24) & $\begin{array}{l}22 \\
(7.51)\end{array}$ & $29(11.24)$ & $\begin{array}{l}21 \\
(8.14)\end{array}$ & 0.011 & 0.234 \\
\hline Renal Failure & $\begin{array}{l}114 \\
(19.35)\end{array}$ & $\begin{array}{l}52 \\
(17.75)\end{array}$ & $44(17.05)$ & $\begin{array}{l}47 \\
(18.22)\end{array}$ & 0.565 & 0.729 \\
\hline Liver Disease & $41(6.96)$ & $\begin{array}{l}14 \\
(4.78)\end{array}$ & $18(6.98)$ & $\begin{array}{l}12 \\
(4.65)\end{array}$ & 0.207 & 0.259 \\
\hline Ulcer & $7(1.19)$ & $3(1.02)$ & $4(1.55)$ & $2(0.78)$ & 0.828 & 0.411 \\
\hline AIDS & $5(0.85)$ & $0(0.00)$ & $4(1.55)$ & $0(0.00)$ & 0.176 & 0.124 \\
\hline Lymphoma & $8(1.36)$ & $6(2.05)$ & $4(1.55)$ & $6(2.33)$ & 0.440 & 0.523 \\
\hline Cancer Metastases & $30(5.09)$ & $\begin{array}{l}16 \\
(5.46)\end{array}$ & $12(4.65)$ & $\begin{array}{l}15 \\
(5.81)\end{array}$ & 0.817 & 0.553 \\
\hline Solid Tumor & $26(4.41)$ & $\begin{array}{l}19 \\
(6.48)\end{array}$ & $9(3.49)$ & $\begin{array}{l}15 \\
(5.81)\end{array}$ & 0.188 & 0.210 \\
\hline Rheumatoid Arthritis & $25(4.24)$ & $8(2.73)$ & $7(2.71)$ & $8(3.10)$ & 0.264 & 0.793 \\
\hline Coagulopathy & $47(7.89)$ & $\begin{array}{l}28 \\
(9.56)\end{array}$ & $26(10.08)$ & $\begin{array}{l}22 \\
(8.53)\end{array}$ & 0.429 & 0.544 \\
\hline Obesity & $57(9.68)$ & $\begin{array}{l}13 \\
(4.44)\end{array}$ & $16(6.20)$ & $\begin{array}{l}13 \\
(5.04)\end{array}$ & 0.007 & 0.566 \\
\hline Weight Loss & $36(6.11)$ & $\begin{array}{l}13 \\
(4.44)\end{array}$ & $12(4.65)$ & $9(3.49)$ & 0.306 & 0.504 \\
\hline $\begin{array}{l}\text { Fluid and Electrolyte } \\
\text { Disorder }\end{array}$ & $\begin{array}{l}282 \\
(47.88)\end{array}$ & $\begin{array}{l}131 \\
(44.71)\end{array}$ & $\begin{array}{l}113 \\
(43.80)\end{array}$ & $\begin{array}{l}114 \\
(44.19)\end{array}$ & 0.375 & 0.929 \\
\hline Blood Loss Anemia & $13(2.21)$ & $\begin{array}{l}15 \\
(5.12)\end{array}$ & $9(3.49)$ & $\begin{array}{l}10 \\
(3.88)\end{array}$ & 0.020 & 0.815 \\
\hline Deficiency Anemia & $29(4.92)$ & $\begin{array}{l}13 \\
(4.44)\end{array}$ & $12(4.65)$ & $\begin{array}{l}10 \\
(3.88)\end{array}$ & 0.749 & 0.663 \\
\hline Alcohol Abuse & $44(7.47)$ & $\begin{array}{l}17 \\
(6.80)\end{array}$ & 13(5.04) & $\begin{array}{l}15 \\
(5.81)\end{array}$ & 0.358 & 0.698 \\
\hline Drug Abuse & $27(4.58)$ & $\begin{array}{l}11 \\
(3.75)\end{array}$ & $10(3.88)$ & $8(3.10)$ & 0.568 & 0.631 \\
\hline
\end{tabular}




\begin{tabular}{|c|c|c|c|c|c|c|}
\hline \multirow{2}{*}{$\begin{array}{l}\text { Variable } \\
\text { Psychoses }\end{array}$} & \multicolumn{2}{|c|}{ Before matching } & \multicolumn{2}{|c|}{ After matching } & \multicolumn{2}{|l|}{$\mathbf{P}$} \\
\hline & $36(6.11)$ & $4(1.37)$ & $14(5.43)$ & $4(1.55)$ & 0.001 & 0.016 \\
\hline Depression & $\begin{array}{l}105 \\
(17.83)\end{array}$ & $\begin{array}{l}20 \\
(6.83)\end{array}$ & $12(4.65)$ & $\begin{array}{l}20 \\
(7.75)\end{array}$ & $<.001$ & 0.144 \\
\hline \multicolumn{7}{|l|}{$\begin{array}{l}\text { Severity of illness [median } \\
(\text { IQR)] }\end{array}$} \\
\hline SOFA score & $3(2,6)$ & $4(2,6)$ & $3(2,6)$ & $4(2,6)$ & 0.002 & 0.269 \\
\hline SAPS II score & $37(29,45)$ & $\begin{array}{l}40 \\
(32,47)\end{array}$ & $38(31,47)$ & $\begin{array}{l}39 \\
(31,46)\end{array}$ & 0.009 & 0.502 \\
\hline SIRS & $3(2,3)$ & $3(2,4)$ & $3(2,4)$ & $3(2,4)$ & 0.003 & 0.606 \\
\hline $\begin{array}{l}\text { Invasive mechanical } \\
\text { ventilation, } \mathrm{n}(\%)\end{array}$ & $\begin{array}{l}220 \\
(37.35)\end{array}$ & $\begin{array}{l}148 \\
(50.51)\end{array}$ & $\begin{array}{l}123 \\
(47.67)\end{array}$ & $\begin{array}{l}119 \\
(46.12)\end{array}$ & $<.001$ & 0.724 \\
\hline \multicolumn{7}{|l|}{ Eosinophils, \% } \\
\hline Eosinophil initial & $\begin{array}{l}0.6 \\
(0.1,1.6)\end{array}$ & $0.2(0,1)$ & $\begin{array}{l}0.4 \\
(0.1,1.3)\end{array}$ & $0.3(0,1)$ & $<.001$ & 0.098 \\
\hline Eosinophil max & $\begin{array}{l}1.0 \\
(0.3,2.3)\end{array}$ & $\begin{array}{l}0.7 \\
(0.2,1.7)\end{array}$ & $\begin{array}{l}0.9 \\
(0.2,2.2)\end{array}$ & $\begin{array}{l}0.7 \\
(0.2,1.9)\end{array}$ & 0.001 & 0.267 \\
\hline Eosinophil mean & $\begin{array}{l}0.6 \\
(0.222,1.6)\end{array}$ & $\begin{array}{l}0.35 \\
(0.1,0.9)\end{array}$ & $\begin{array}{l}0.5 \\
(0.15,1.33)\end{array}$ & $\begin{array}{l}0.35 \\
(0.1,1.0)\end{array}$ & $<.001$ & 0.031 \\
\hline Hospital LOS, day & $7(4,12)$ & $9(5,17)$ & $8(5,13)$ & $9(5,17)$ & $<.001$ & 0.067 \\
\hline 30-day mortality & $\begin{array}{l}118 \\
(20.03)\end{array}$ & $\begin{array}{l}82 \\
(27.99)\end{array}$ & $59(22.87)$ & $\begin{array}{l}69 \\
(26.74)\end{array}$ & 0.010 & 0.359 \\
\hline $\begin{array}{l}\text { Hospital admission times, } \\
\text { mean (min, max) }\end{array}$ & $\begin{array}{l}1.312 \\
(1,19)\end{array}$ & $\begin{array}{l}1.235 \\
(1,10)\end{array}$ & $\begin{array}{l}1.442 \\
(1,19)\end{array}$ & $\begin{array}{l}1.213 \\
(1,10)\end{array}$ & 0.140 & 0.059 \\
\hline Multiple admissions & $\begin{array}{l}110 \\
(18.68)\end{array}$ & $\begin{array}{l}43 \\
(14.68)\end{array}$ & $53(20.54)$ & $\begin{array}{l}38 \\
(14.73)\end{array}$ & 0.140 & 0.083 \\
\hline
\end{tabular}

IQR interquartile range, SOFA sequential organ failure assessment, SAPSII Simplified Acute Physiology Score II, SIRS systemic inflammatory response syndrome, LOS length of stay

The results of multivariate logistic regression analyses for primary outcome and secondary outcomes are shown in Table 3 and Fig. 2. In multivariate model 1, covariates were adjusted for age (years), Elixhauser comorbidity score, SAPS II, SIRS, invasive mechanical ventilation, and initial value of eosinophil concentration (Supplementary Table 1). In model 2, covariates were adjusted for age (years), cardiac arrhythmias, liver disease, metastatic cancer, solid tumor, coagulopathy, obesity, drug abuse and psychoses, SOFA, SIRS, mechanical ventilation, and initial value of eosinophil concentration (Supplementary Table 2). The multivariate logistic regression analyses showed there was no statistically 
significant difference between patients treated with systemic corticosteroids and the control group with regard to the main end-point, namely 30-day mortality in this study cohort, as well as in-hospital mortality. The adjusted odds ratio (OR) of 30-day mortality for systemic corticosteroid treatment was 1.296 (95\% Cl: $0.907-1.852, p=0.155)$ in model 1 and 1.243 (95\% Cl: 0.867-1.782, $p=0.236)$ in model 2. However, a significant negative effect of systemic corticosteroid treatment in terms of hospital length of stay was found from the multivariate logistic regression analyses (Fig. 2) with an adjusted odds ratio of 1.583 (95\% Cl: $1.160-2.162 ; p=0.004)$ in model 1 and 1.561 (95\% Cl: $1.133-2.149 ; p=0.006)$ in model 2.

Table 3

Association between systemic corticosteroids treatment and outcomes using multivariate logistic regression analyses

\begin{tabular}{|lllllllll|}
\hline Outcomes & \multicolumn{3}{l}{ Multivariate Model 1 } & \multicolumn{5}{l|}{ Multivariate Model 2 } \\
\hline & OR & $95 \% \mathrm{Cl}$ & & $\mathrm{P}$ & $\mathrm{OR}$ & $95 \% \mathrm{Cl}$ & $\mathrm{p}$ \\
\hline 30-day mortality & 1.296 & 0.907 & 1.852 & 0.155 & 1.243 & 0.867 & 1.782 & 0.236 \\
\hline In-hospital mortality & 1.466 & 0.986 & 2.180 & 0.059 & 1.398 & 0.936 & 2.089 & 0.102 \\
\hline Hospital LOS (> 8days) & 1.583 & 1.160 & 2.162 & 0.004 & 1.561 & 1.133 & 2.149 & 0.006 \\
\hline
\end{tabular}

Multivariate model 1: adjusted for age (years), Elixhauser comorbidity score, SAPS II, SIRS, invasive mechanical ventilation, and initial value of eosinophil concentration, and mean VIF was 1.09 for 30-day mortality. Multivariate model 2: adjusted for age (years), SOFA, SIRS, invasive mechanical ventilation, and initial value of eosinophil concentration, cardiac arrhythmias, liver disease, metastatic cancer, solid tumor, coagulopathy, obesity, drug abuse and psychoses, and mean VIF was 1.07 for 30-day mortality.

\section{Subgroups Analyses}

Association of GC use and outcomes in subgroups by using multivariate logistic regression analysis is shown in Table 4. The results showed that systemic corticosteroid treatment may be a risk factor for death in hospital in the subgroup with EOS initial concentration $\geq 2 \%$ (OR 6.645; 95\% Cl 1.537-28.723; $p$ $=0.011$ ). Meanwhile, as shown in Table 4, there was no difference regarding mortality in the steroidtreated and control groups in the subgroup of patients with initial eosinophil concentration $<0.35 \%$. In the subgroup of patients with initial eosinophil concentration $\geq 0.35 \%$, corticosteroid treatment resulted in worse outcomes with the adjusted odds ratio 1.691 (95\% Cl: $1.002-2.855, \mathrm{p}=0.049)$ and 2.247 (95\% Cl: $1.218-4.147, p=0.010$ ) of 30-day mortality and in-hospital mortality respectively. 
Table 4

Association of GC use and outcomes in subgroups using multivariate logistic regression analyses

\begin{tabular}{|c|c|c|c|c|c|}
\hline Subgroup & Outcomes & OR & $95 \% \mathrm{Cl}$ & & $\mathbf{P}$ \\
\hline & 30-day mortality & 1.018 & 0.622 & 1.666 & 0.944 \\
\hline \multirow[t]{3}{*}{ EOS < $0.35(n=404)$} & In-hospital mortality & 1.107 & 0.656 & 1.867 & 0.703 \\
\hline & Hospital LOS (> 8days) & 1.664 & 1.068 & 2.593 & 0.024 \\
\hline & 30-day mortality & 1.691 & 1.002 & 2.855 & 0.049 \\
\hline \multirow[t]{3}{*}{$E O S \geq 0.35(n=478)$} & In-hospital mortality & 2.247 & 1.218 & 4.147 & 0.010 \\
\hline & Hospital LOS (> 8days) & 1.555 & 1.000 & 2.417 & 0.050 \\
\hline & 30-day mortality & 1.252 & 0.859 & 1.824 & 0.243 \\
\hline \multirow[t]{3}{*}{ EOS $<2(n=727)$} & In-hospital mortality & 1.370 & 0.905 & 2.074 & 0.136 \\
\hline & Hospital LOS (> 8days) & 1.531 & 1.094 & 2.144 & 0.013 \\
\hline & 30-day mortality & 2.718 & 0.930 & 7.945 & 0.068 \\
\hline \multirow[t]{2}{*}{$E O S \geq 2(n=155)$} & In-hospital mortality & 6.645 & 1.537 & 28.723 & 0.011 \\
\hline & Hospital LOS (> 8days) & 2.055 & 0.910 & 4.641 & 0.083 \\
\hline
\end{tabular}

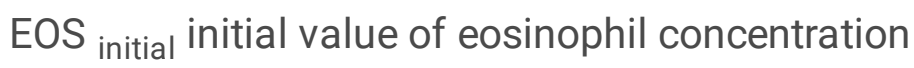

The logistic regression was adjusted for age (years), Elixhauser comorbidity score, SAPS II, SIRS, invasive mechanical ventilation.

In this study, intravenous administration did not significantly affect 30-day mortality compared to oral administration $(p=0.101)$. However, a significantly negative effect on 30-day and in-hospital mortality was observed when comparing intravenous administration to co-administration method (intravenous administration and oral administration) as shown in Table 5. 
Table 5

Association of the route of administration and outcomes in GC group

\begin{tabular}{|llllll|}
\hline Comparison & Outcomes & OR & $95 \% \mathrm{Cl}$ & $\mathrm{P}$ \\
\hline & 30-day mortality & 2.252 & 0.834 & 6.075 & 0.109 \\
\hline PO $(\mathrm{n}=59)$ vs IV $(\mathrm{n}=64)$ & In-hospital mortality & 3.021 & 1.009 & 9.044 & 0.048 \\
\hline & Hospital LOS (> 8days) & 1.326 & 0.559 & 3.145 & 0.521 \\
\hline & 30-day mortality & 1.194 & 0.503 & 2.832 & 0.687 \\
\hline Combination $(\mathrm{n}=170)$ vs PO & In-hospital mortality & 0.818 & 0.293 & 2.284 & 0.701 \\
\hline & Hospital LOS (> 8days) & 0.442 & 0.228 & 0.855 & 0.015 \\
\hline & 30-day mortality & 1.432 & 1.019 & 2.013 & 0.039 \\
\hline Combination $(\mathrm{n}=170)$ vs IV & In-hospital mortality & 1.460 & 1.027 & 2.075 & 0.035 \\
\hline & Hospital LOS (> 8days $)$ & 0.726 & 0.528 & 0.997 & 0.048 \\
\hline
\end{tabular}

PO oral administration, IV intravenous administration

The logistic regression was adjusted for age (years), Elixhauser comorbidity score, SAPS II, SIRS, invasive mechanical ventilation, initial value of eosinophil concentration.

\section{Propensity Score Analysis}

The 258 patients who did not received systemic corticosteroid treatment were matched to 258 patients who received systemic corticosteroid treatment by PSM. After PSM, the imbalance in the covariates between the non-GC and GC groups was significantly minimized (Table 2). Since there were still residual imbalances between GC and non-GC groups, multivariate logistic regression was then used. As shown in Table 6, systemic corticosteroid treatment was not associated with improved 30-day mortality in overall population $(\mathrm{OR}=1.144,95 \% \mathrm{Cl}: 0.744-1.759 ; \mathrm{p}=0.541)$. However, the adjusted OR showed a close to statistically adverse effect on hospital LOS when comparing the GC group to non-GC group (OR $=1.395$, $95 \% \mathrm{Cl}: 0.964-2.019 ; \mathrm{p}=0.077$ ) (Table 6). Variable importance with respect to 30 -day mortality is shown in Supplementary Table 3. 
Table 6

ORs of systemic corticosteroids treatment for outcomes after propensity score matching

\begin{tabular}{|lllll|}
\hline Outcomes & OR & $\mathbf{9 5 \% C l}$ & & $\mathbf{P}$ \\
\hline 30-day mortality & 1.144 & 0.744 & 1.759 & 0.541 \\
\hline In-hospital mortality & 1.281 & 0.796 & 2.063 & 0.308 \\
\hline Hospital LOS (>8days) & 1.395 & 0.964 & 2.019 & 0.077 \\
\hline
\end{tabular}

The logistic regression was adjusted for SAPS II, SIRS, invasive mechanical ventilation, mean value of eosinophil concentration and psychoses.

\section{Discussion}

In this retrospective cohort study based on a large critical care database MIMIC-III (v1.4), we found that systemic corticosteroid treatment did not decrease 30-day mortality or shorten hospital length of stay in critically ill patients with AECOPD in ICU.

Biologically, glucocorticoids can relieve airways inflammation and in return reduce the airways resistance to improve the dyspnea and the work of breathing [17]. The use of systemic glucocorticoids has been recommended during COPD exacerbations by GOLD 2021 (https://goldcopd.org/2021-gold-reports/). One strong available evidence is a cochrane meta-analysis which summarized the efficacy of systemic corticosteroid therapy on the outcomes of AECOPD [5]. The results of this review showed beneficial effects of systemic corticosteroid treatment on treatment failure, relapse by one month and shortening length of stay in AECOPD hospital inpatients not requiring assisted ventilation outside ICU. Meanwhile, 30-day mortality was not reduced by treatment with systemic corticosteroid compared with placebo. However, it is important to realize that treatments developed outside the ICU may not prove effective in the ICU and even if such approaches provide benefit for critically ill patients, the risk-benefit calculus may be different [18].

There have been two researches conducted double-blind, multicenter, placebo-controlled randomized trials comparing corticosteroids with placebo to document the value of these agents in the treatment of severe AECOPDs requiring ventilatory support in ICU. However, the results of these two studies are contradictory. Alia et al. [6] reported a significant reduction in the median duration of mechanical ventilation ( 3 days vs 4 days; $p=0.04$ ), a trend toward a shorter median length of ICU stay ( 6 days vs 7 days; $p=0.09)$, and significant reduction in the rate of NIV failure ( $0 \%$ vs $37 \% ; p=0.04)$ associated with corticosteroid treatment. Abroug et al. [7] observed similar median mechanical ventilation duration (6 days vs 6 days; $p=0.87$ ), similar intensive care unit length of stay ( 9 days vs 8 days; $p=0.88$ ) and no statistical difference NIV failure rate $(15.7 \%$ vs $12.7 \% ; p=0.59)$ between the steroid-treated and control group. The different results may be explained by the different routes of corticosteroid steroid used in the studies and the severity of AECOPD (more severe respiratory acidosis and hypercapnia in the latter). 
Combining these two studies, the review by Walters et al. [5] drew the conclusion that there was no difference in length of ICU stay and the duration of assisted ventilation for corticosteroid treatment compared with the control for AECOPD patients requiring assisted ventilation in the ICU setting.

Short-term administration of systemic glucocorticoids may cause secondary infections, hyperglycemia, and a range of mood and behavioral changes [19]. Adverse effects of the long-term therapy include osteoporosis, hypertension, myopathy, and adrenal insufficiency [19-22]. Increased risk of hyperglycemia requiring treatment following systemic glucocorticoid usage was both reported by Alia et al. [6] and Abroug et al. [7]. The risk-benefit balance of systemic steroids is negative from the results of the study by Abroug et al. [7]. In this study, we observed no statistically significant difference in 30-day mortality and in-hospital mortality between systemic corticosteroid group and the control group (Table 3 and Fig. 2). In addition, patients received systemic corticosteroid treatment had a greater chance of being hospitalized for more than eight days from the multivariate logistic regression (Table 3 and Fig. 2). The result was similar after PSM (Table 6). Taken the side effects into consideration, we recommend that the systematic administration of corticosteroids in severe COPD exacerbation in ICU needs to be cautious.

Some randomized studies showed that therapy with oral prednisolone is equally effective to intravenous administration of glucocorticoids [23,24]. A pharmacoepidemiological cohort study conducted at 414 US hospitals involving almost 80,000 AECOPD patients to a non-intensive care setting demonstrated that the risk of treatment failure (in-hospital mortality, initiation of mechanical ventilation, or readmission for AECOPD within 30 days of discharge) among patients treated with low doses of oral steroids was not worse than for those treated with high dose intravenous therapy [25]. However, the study regarding the route of steroid administration with AECOPDs in ICU has not been carried out. In this cohort study, oral administration presented similar efficacy with intravenous and the combined administration, with no difference in 30-day mortality and hospital LOS (> 8days). Besides, patients receiving intravenous steroids had higher risk of death within 30 days or in hospital, as well as a greater probability to stay in hospital for more than 8 days compared to the combined administration. Real-world information reminds us that studies with regard to steroids treatment should not be limited to the comparison of oral and intravenous administration, the co-administration method should also be included.

Eosinophil levels may be a helpful marker to predict outcomes in AECOPD and to direct corticosteroid therapy during exacerbations [26-29]. Singh et al. [30] assessed the prevalence of eosinophilic inflammation in COPD subjects and found that $37 \%$ of the COPD subjects had blood eosinophil counts persistently $\geq 2 \%$. However, the prevalence of eosinophilic inflammation in COPD subjects requiring ICU admission has not been well defined. In this cohort study, only $17.57 \%$ of the subjects with a COPD exacerbation requiring ICU had a peripheral blood initial eosinophil concentration greater than $2 \%$ and the median of initial blood eosinophil concentrations was $0.4 \%$ which was lower than other researches [3133] aimed at AECOPD. A previous study drew the receiver operating characteristic (ROC) curve for the prediction of in-hospital mortality by eosinophil concentrations in hospital admissions diagnosed with AECOPD in MIMIC-III v1.4 database [34]. The area under the ROC curve for initial eosinophil concentration 
was 0.608 and the discriminatory eosinophil thresholds were $0.35 \%$ (sensitivity $=0.59$, specificity $=0.61$ ) for in-hospital mortality [34]. Thus, the cut-off value $0.35 \%$ was also used to distinguish subgroups.

Lower-eosinophilic patients were found to experienced poorer clinical outcomes in a prospective, multicenter, observational cohort study [31]. In addition, a retrospective observational cohort study showed that COPD exacerbations with acute respiratory failure requiring ICU admission had a shorter median length of ICU stay and lower mortality with a peripheral eosinophil level $>2 \%$ [24]. Similar results appeared in our research. As shown in Supplementary Table 1, increased blood eosinophil level was associated with decreased 30-day mortality in our study $(\mathrm{OR}=0.688,95 \% \mathrm{Cl}: 0.688-0.946, p=0.008)$.

Targeting corticosteroid therapy in a subgroup of exacerbations dependent on the peripheral eosinophil count may be helpful to reduce inappropriate use of systemic corticosteroids [35]. Recent studies suggested that glucocorticoids may be more efficacious to treat acute COPD exacerbations in patients with higher levels of blood eosinophils ( $\geq 2 \%$ ) [26-29]. However, things seem to be different in ICU. Little evidence was found that systemic corticosteroids provided benefit in patients with a blood eosinophil initial concentration $<2 \%$ in our study Table 4 . What's more, in the subgroup with a blood eosinophil initial concentration $\geq 2 \%$, patients treated with systemic corticosteroids had a significantly increased inhospital mortality compared with non-GC group $(\mathrm{OR}=6.645,95 \% \mathrm{Cl}: 1.537-28.723, \mathrm{p}=0.011)$. When the cut-off value set to $0.35 \%$, the effect of eosinophil level on the efficacy of glucocorticoids on the mortality of severe AECOPD patients was more apparent. As shown in Table 4, there was no difference regarding 30-day mortality and in-hospital mortality in the steroid-treated and control groups in the subgroup of patients with initial eosinophil concentration $<0.35 \%$. Critically, in the subgroup of patients with initial eosinophil concentration $\geq 0.35 \%$, corticosteroid treatment resulted in worse outcomes compared with non-GC group. The adjusted odds ratio of 30-day mortality and in-hospital mortality for glucocorticoid therapy were $1.691(95 \% \mathrm{Cl}: 1.002-2.855, \mathrm{p}=0.049)$ and $2.247(95 \% \mathrm{Cl}: 1.218-4.147, \mathrm{p}=0.010)$ respectively.

Therefore, recommendation of systemic corticosteroid treatment is not supported in critically severe AECOPD with a high blood eosinophil initial concentration in ICU by these results until a more precise evidence emerges. More researches are needed to explore the cut-off value for eosinophils and the reasons for the conflicting results between ICU patients and non-ICU patients.

Although this study was relatively considerable sample size, it has several limitations. First, our research is a database-based retrospective single-center study. The inherent bias could not be avoided. Outcomes in this study only included 30-day mortality, in-hospital mortality and hospital LOS (> 8days), which were not as flexible as randomized controlled trials. Second, systemic steroids use in the 30 days prior to hospital admission was unknown. This may cause some bias in the results though it is closer to the real world. Third, only blood eosinophil concentrations were considered in this study because the numeric value of the blood eosinophil counts was hugely lacked in the MIMIC-III database. The subgroup analysis will be more complete if blood eosinophil counts were considered together. 


\section{Conclusion}

In this cohort study, our results showed that systemic corticosteroid therapy was not associated with improved mortality in critically ill patients with acute exacerbations of chronic obstructive pulmonary disease. Patients who have received glucocorticoid therapy with AECOPD in the ICU have a higher probability of being hospitalized for more than eight days. In the subgroup of critically ill patients with high blood eosinophil concentration, corticosteroid treatment resulted in worse outcomes compared with non-GC group. Administration of systemic steroids in severe AECOPDs in ICU should be very cautious, especially for patients with high eosinophils. Further randomized controlled trials are needed to confirm these results.

\section{Abbreviations}

ICU: Intensive care unit; AECOPD: Acute exacerbation of chronic obstructive pulmonary disease; MIMIC-III: Medical Information Mart for Intensive Care III; PSM: Propensity score matching; GC: Glucocorticoid; OR: Odds ratio; COPD: Chronic obstructive pulmonary disease; NIV: Noninvasive ventilation; EOS: Eosinophil; IRB: institutional review board; SOFA: Sequential organ failure assessment; SAPS II: Simplified acute physiology score II; SIRS: Systemic inflammatory response syndrome; LOS: Length of stay; PS: Propensity score; PO: Oral administration; IV: Intravenous administration

\section{Declarations}

\section{Ethics approval and consent to participate}

The Institutional review boards at the Beth Israel Deaconess Medical Center (protocol 2001-P-001699/14) and Massachusetts Institute of Technology (protocol 0403000206) have approved the data collection and the use of MIMIC-III for research purposes and granted waiver of informed consent. This study is reported following the STrengthening the Reporting of OBservational studies in Epidemiology (STROBE) statement.

\section{Consent for publication}

Not applicable

\section{Availability of data and materials}

The data used for this study can be accessed from the MIMIC-III database (https://mimic.physionet.org/).

\section{Competing interests}

The authors declare that they have no competing interests. 


\section{Funding}

This study was supported by the National Nature Science Foundation of China (NO. 82074358).

\section{Authors' contributions}

XMZ, HLH and SC contributed to the conception of the study and interpretation of findings. TYP performed the initial data extraction. TYP, LB, and KJZ conducted the statistical analyses. TYP, JW, YHL and WZZ were major contributors in writing the manuscript. All authors read and approved the final manuscript.

\section{Acknowledgements}

The authors would like to thank the Beth Israel Deaconess Medical Center and the team of the Laboratory for Computational Physiology from the Massachusetts Institute of Technology (LCP-MIT) for the project of Medical Information Mart for Intensive Care III database (MIMIC III).

\section{Author details}

${ }^{1}$ Affiliated Hospital of Nanjing University of Chinese Medicine, Nanjing, China. ${ }^{2}$ Department of Respiratory Medicine, Jiangsu Province Hospital of Chinese Medicine, Nanjing, China. ${ }^{3}$ School of Mathematical Science, Shanghai Jiao Tong University, Shanghai, China.

\section{References}

1. Collaborators GBDCoD. Global, regional, and national age-sex-specific mortality for 282 causes of death in 195 countries and territories, 1980-2017: a systematic analysis for the Global Burden of Disease Study 2017. Lancet. 2018;392:1736-88. doi:10.1016/S0140-6736(18)32203-7.

2. Donaldson GC, Seemungal TA, Bhowmik A, Wedzicha JA. Relationship between exacerbation frequency and lung function decline in chronic obstructive pulmonary disease. Thorax. 2002;57:847-52. doi:10.1136/thorax.57.10.847.

3. Donaldson GC, Wilkinson TM, Hurst JR, Perera WR, Wedzicha JA. Exacerbations and time spent outdoors in chronic obstructive pulmonary disease. Am J Respir Crit Care Med. 2005;171:446-52. doi:10.1164/rccm.200408-10540C.

4. Soler-Cataluna JJ, Martinez-Garcia MA, Roman Sanchez P, Salcedo E, Navarro M, Ochando R. Severe acute exacerbations and mortality in patients with chronic obstructive pulmonary disease. Thorax. 2005;60:925-31. doi:10.1136/thx.2005.040527.

5. Walters JA, Tan DJ, White CJ, Gibson PG, Wood-Baker R, Walters EH. Systemic corticosteroids for acute exacerbations of chronic obstructive pulmonary disease. Cochrane Database Syst Rev. 2014:CD001288. doi:10.1002/14651858.CD001288.pub4. 
6. Alia I, de la Cal MA, Esteban A, Abella A, Ferrer R, Molina FJ, et al. Efficacy of corticosteroid therapy in patients with an acute exacerbation of chronic obstructive pulmonary disease receiving ventilatory support. Arch Intern Med. 2011;171:1939-46. doi:10.1001/archinternmed.2011.530.

7. Abroug F, Ouanes-Besbes L, Fkih-Hassen M, Ouanes I, Ayed S, Dachraoui F, et al. Prednisone in COPD exacerbation requiring ventilatory support: an open-label randomised evaluation. Eur Respir J. 2014;43:717-24. doi:10.1183/09031936.00002913.

8. Johnson AE, Pollard TJ, Shen L, Lehman LW, Feng M, Ghassemi M, et al. MIMIC-III, a freely accessible critical care database. Sci Data. 2016;3:160035. doi:10.1038/sdata.2016.35.

9. Johnson AE, Stone DJ, Celi LA, Pollard TJ. The MIMIC Code Repository: enabling reproducibility in critical care research. J Am Med Inform Assoc. 2018;25:32-9. doi:10.1093/jamia/ocx084.

10. Wedzicha JA, Seemungal TA. COPD exacerbations: defining their cause and prevention. Lancet. 2007;370:786-96. doi:10.1016/S0140-6736(07)61382-8.

11. Jain SS, Sarkar IN, Stey PC, Anand RS, Biron DR, Chen ES. Using Demographic Factors and Comorbidities to Develop a Predictive Model for ICU Mortality in Patients with Acute Exacerbation COPD. AMIA Annu Symp Proc. 2018;2018:1319-28.

12. Vincent JL, Moreno R, Takala J, Willatts S, De Mendonca A, Bruining H, et al. The SOFA (Sepsisrelated Organ Failure Assessment) score to describe organ dysfunction/failure. On behalf of the Working Group on Sepsis-Related Problems of the European Society of Intensive Care Medicine. Intensive Care Med. 1996;22:707-10. doi:10.1007/BF01709751.

13. Le Gall JR, Lemeshow S, Saulnier F. A new Simplified Acute Physiology Score (SAPS II) based on a European/North American multicenter study. JAMA. 1993;270:2957-63. doi:10.1001/jama.270.24.2957.

14. American College of Chest Physicians/Society of Critical Care Medicine Consensus Conference: definitions for sepsis and organ failure and guidelines for the use of innovative therapies in sepsis. Crit Care Med. 1992;20:864 - 74.

15. Elixhauser A, Steiner C, Harris DR, Coffey RM. Comorbidity measures for use with administrative data. Med Care. 1998;36:8-27. doi:10.1097/00005650-199801000-00004.

16. Quan H, Sundararajan V, Halfon P, Fong A, Burnand B, Luthi JC, et al. Coding algorithms for defining comorbidities in ICD-9-CM and ICD-10 administrative data. Med Care. 2005;43:1130-9. doi:10.1097/01.mlr.0000182534.19832.83.

17. Vasques F, Camporota L, Barrett NA. Nonantibiotic Pharmacological Treatment of Severe Chronic Obstructive Pulmonary Disease Exacerbations. Semin Respir Crit Care Med. 2020;41:842-50. doi:10.1055/s-0040-1714379.

18. Shorr AF, Chan CM. Clinical trials in the critically ill: practical and ethical challenges: comment on "Efficacy of corticosteroid therapy in patients with an acute exacerbation of chronic obstructive pulmonary disease receiving ventilatory support". Arch Intern Med. 2011;171:1946-7. doi:10.1001/archinternmed.2011.531. 
19. McEvoy CE, Niewoehner DE. Adverse effects of corticosteroid therapy for COPD. A critical review. Chest. 1997;111:732-43. doi:10.1378/chest.111.3.732.

20. Decramer M, Stas KJ. Corticosteroid-induced myopathy involving respiratory muscles in patients with chronic obstructive pulmonary disease or asthma. Am Rev Respir Dis. 1992;146:800-2. doi:10.1164/ajrccm/146.3.800.

21. Decramer M, Lacquet LM, Fagard R, Rogiers P. Corticosteroids contribute to muscle weakness in chronic airflow obstruction. Am J Respir Crit Care Med. 1994;150:11-6.

doi:10.1164/ajrccm.150.1.8025735.

22. Walsh LJ, Wong CA, Oborne J, Cooper S, Lewis SA, Pringle M, et al. Adverse effects of oral corticosteroids in relation to dose in patients with lung disease. Thorax. 2001;56:279-84. doi:10.1136/thorax.56.4.279.

23. de Jong YP, Uil SM, Grotjohan HP, Postma DS, Kerstjens HA, van den Berg JW. Oral or IV prednisolone in the treatment of COPD exacerbations: a randomized, controlled, double-blind study. Chest. 2007;132:1741-7. doi:10.1378/chest.07-0208.

24. Ceviker Y, Sayiner A. Comparison of two systemic steroid regimens for the treatment of COPD exacerbations. Pulm Pharmacol Ther. 2014;27:179-83. doi:10.1016/j.pupt.2013.03.004.

25. Lindenauer PK, Pekow PS, Lahti MC, Lee Y, Benjamin EM, Rothberg MB. Association of corticosteroid dose and route of administration with risk of treatment failure in acute exacerbation of chronic obstructive pulmonary disease. JAMA. 2010;303:2359-67. doi:10.1001/jama.2010.796.

26. Hurst JR, Vestbo J, Anzueto A, Locantore N, Mullerova H, Tal-Singer R, et al. Susceptibility to exacerbation in chronic obstructive pulmonary disease. N Engl J Med. 2010;363:1128-38. doi:10.1056/NEJMoa0909883.

27. Bafadhel M, McKenna S, Terry S, Mistry V, Pancholi M, Venge P, et al. Blood eosinophils to direct corticosteroid treatment of exacerbations of chronic obstructive pulmonary disease: a randomized placebo-controlled trial. Am J Respir Crit Care Med. 2012;186:48-55. doi:10.1164/rccm.201108$15530 \mathrm{C}$.

28. Bafadhel M, McKenna S, Terry S, Mistry V, Reid C, Haldar P, et al. Acute exacerbations of chronic obstructive pulmonary disease: identification of biologic clusters and their biomarkers. Am J Respir Crit Care Med. 2011;184:662-71. doi:10.1164/rccm.201104-05970C.

29. Sivapalan P, Lapperre TS, Janner J, Laub RR, Moberg M, Bech CS, et al. Eosinophil-guided corticosteroid therapy in patients admitted to hospital with COPD exacerbation (CORTICO-COP): a multicentre, randomised, controlled, open-label, non-inferiority trial. Lancet Respir Med. 2019;7:699709. doi:10.1016/S2213-2600(19)30176-6.

30. Singh D, Kolsum U, Brightling CE, Locantore N, Agusti A, Tal-Singer R, et al. Eosinophilic inflammation in COPD: prevalence and clinical characteristics. Eur Respir J. 2014;44:1697-700. doi:10.1183/09031936.00162414.

31. Wu HX, Zhuo KQ, Cheng DY. Peripheral Blood Eosinophil as a Biomarker in Outcomes of Acute Exacerbation of Chronic Obstructive Pulmonary Disease. Int J Chron Obstruct Pulmon Dis. 
2019;14:3003-15. doi:10.2147/COPD.S226783.

32. Gao J, Zhang M, Zhou L, Yang X, Wu H, Zhang J, et al. Correlation between fractional exhaled nitric oxide and sputum eosinophilia in exacerbations of COPD. Int $\mathrm{J}$ Chron Obstruct Pulmon Dis. 2017;12:1287-93. doi:10.2147/COPD.S134998.

33. Ko FWS, Chan KP, Ngai J, Ng SS, Yip WH, Ip A, et al. Blood eosinophil count as a predictor of hospital length of stay in COPD exacerbations. Respirology. 2020;25:259-66. doi:10.1111/resp.13660.

34. Yang J, Yang J. Association Between Blood Eosinophils and Mortality in Critically III Patients with Acute Exacerbation of Chronic Obstructive Pulmonary Disease: A Retrospective Cohort Study. Int J Chron Obstruct Pulmon Dis. 2021;16:281-8. doi:10.2147/COPD.S289920.

35. Bafadhel M, Davies L, Calverley PM, Aaron SD, Brightling CE, Pavord ID. Blood eosinophil guided prednisolone therapy for exacerbations of COPD: a further analysis. Eur Respir J. 2014;44:789-91. doi:10.1183/09031936.00062614.

\section{Figures}

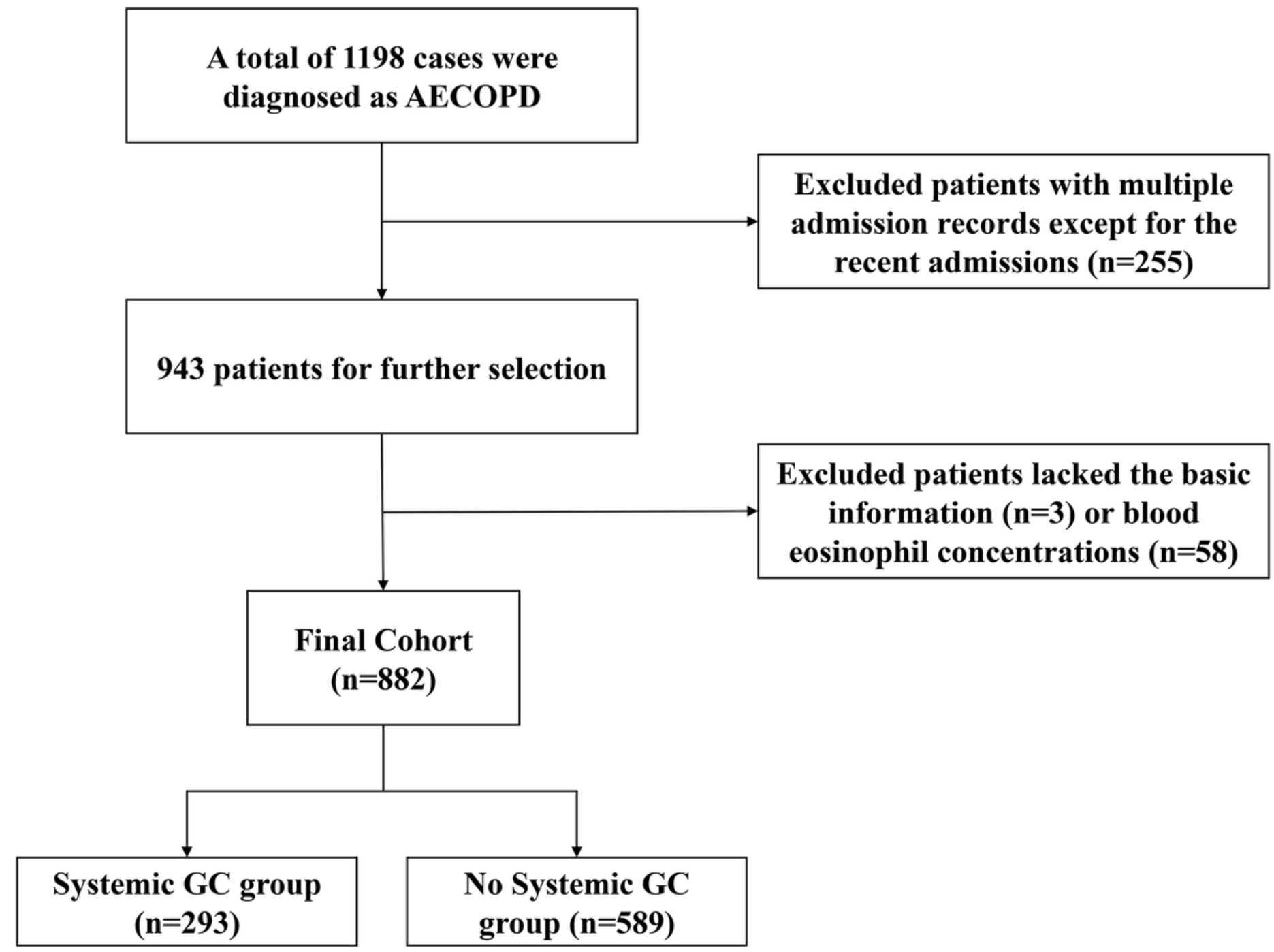


Figure 1

Flow chart of patient selection

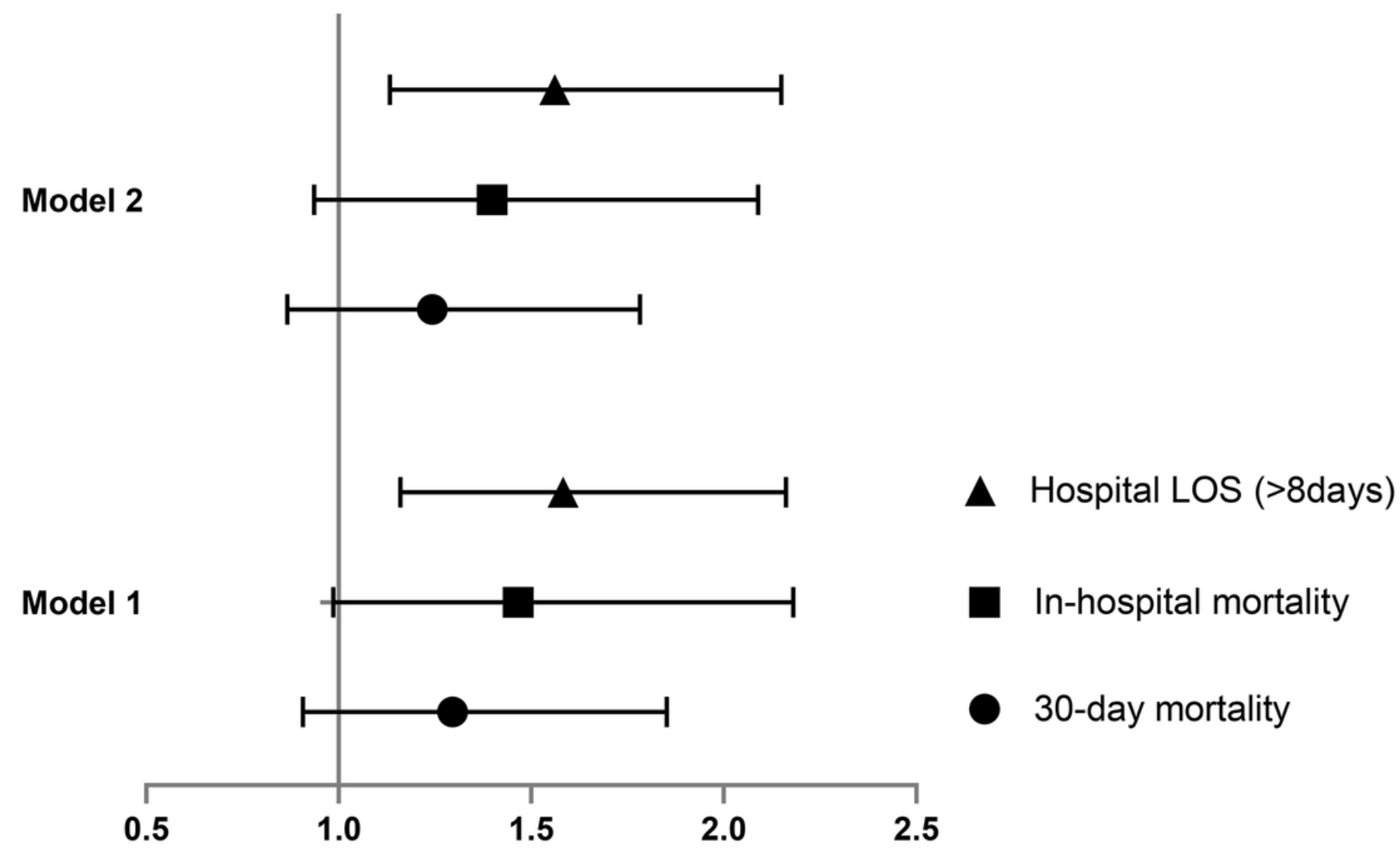

Figure 2

Forest plot showing the effect of systemic corticosteroid treatment on outcomes

\section{Supplementary Files}

This is a list of supplementary files associated with this preprint. Click to download.

- Supplementarymaterials.docx 